\title{
Detecting Cryptosporidium in Stool Samples Submitted to a Reference Laboratory
}

\author{
Kimberly Mergen, ${ }^{1}$ Noel Espina, ${ }^{1}$ Allen Teal, ${ }^{1}$ and Susan Madison-Antenucci ${ }^{1,2 \star}$ \\ ${ }^{1}$ Parasitology Laboratory, Wadsworth Center, NYSDOH, Albany, New York; ${ }^{2}$ School of Public Health, Biomedical Sciences, \\ University at Albany, Albany, New York
}

\begin{abstract}
When considering methods of detecting Cryptosporidium in patient samples, clinical and public health laboratories have historically relied primarily on microscopy. However, microscopy is time intensive and requires trained personnel to accurately identify pathogens that are present. Even with skilled analysts, the parasitemia level has the potential to fall below the level of detection. In addition, public health laboratories do not always receive specimens in fixatives that are compatible with the desired microscopic method. Antigen-based and molecular methods have proven to be effective at identifying Cryptosporidium at low levels and require less training and hands-on time. Here, we have developed and validated a real-time polymerase chain reaction (RT-PCR) laboratory-developed test (LDT) that identifies Cryptosporidium hominis and Cryptosporidium parvum, and also includes detection at the genus level to identify additional species that occasionally cause disease in humans. Results of the molecular test were compared with those obtained from modified acid-fast microscopy, immunofluorescent microscopy, an antigen-based detection rapid test, and a commercial gastrointestinal panel (Gl panel). Of 40 positive samples, microscopy and antigen-based methods were able to detect Cryptosporidium in only 20 and 21 samples, respectively. The Gl panel detected 33 of the 40 positive samples, even though not all specimens were received in the recommended preservative. The LDT detected Cryptosporidium in all 40 positive samples. When comparing each method for the detection of Cryptosporidium, our results indicate the LDT is an accurate, reliable, and cost-effective method for a clinical public health reference laboratory.
\end{abstract}

\section{INTRODUCTION}

The protozoon parasite Cryptosporidium causes the intestinal disease cryptosporidiosis in humans. Cryptosporidiosis symptoms typically include watery diarrhea, dehydration, and weight loss, and may last for up to 2 weeks. Infections are spread by consuming or having contact with contaminated water, consuming contaminated food, or close contact with people or animals that are infected. In North America, swimming pools and other recreational waters are common sources of infection for multiple reasons; the oocysts are passed in large numbers by infected individuals, are immediately infectious, hardy in the environment, insensitive to chlorine levels maintained in pools, too small (4-6 $\mu \mathrm{m}$ diameter) to be removed by typical filtration systems, and are able to cause an infection when as few as 10 organisms are ingested. ${ }^{1,2}$

In 2014, 8,682 cases of cryptosporidiosis were reported to the CDC. However, diagnosis is likely to be significantly underreported, and estimates of the number of actual episodes of illness due to Cryptosporidium in the United States are as high as $748,000{ }^{3,4}$ From 1995 to 2004 , the average incidence was slightly more than one case per 100,000 people, with a range of 0.9-1.4. In 2005, however, this number started to increase following a jump in the number of reported cases, leading to a new higher steady-state incidence rate of almost three cases per 100,000 people. ${ }^{5}$ Examples of contact with contaminated water that contributed to the increase in reported cases include an outbreak in a New York spray park in $2005,{ }^{6}$ contaminated swimming pools in Utah, ${ }^{7}$ a manmade lake in Texas in 2008, ${ }^{8}$ and contaminated drinking water in a municipality in Oregon in $2013,{ }^{9}$ which sickened $28 \%$ of the community. Increased awareness due to outbreaks such as these, as well as advances in testing methods, could have

\footnotetext{
*Address correspondence to Susan Madison-Antenucci, Parasitology Laboratory, Wadsworth Center, NYSDOH, 120 New Scotland Ave., Rm. 3112, Albany, NY 12201-2002. E-mail: s.antenucci@ health.ny.gov
}

also contributed to the higher reported incidence rates. Given that only a small percentage of cryptosporidiosis cases are believed to be reported, efforts to detect outbreaks earlier are likely to benefit from greater awareness of and more sensitive testing for this intestinal parasite.

Diagnosis by microscopic examination of acid-fast stained slides has been the gold standard, and although it is inexpensive, it is also labor intensive and lacks sensitivity compared with other methods. Immunofluorescent microscopy is more sensitive but still labor intensive. Antigen detection is simple to perform using rapid diagnostic tests, but their performance has been variable. ${ }^{10-13}$ More recently, FDAapproved multiplex panels that detect a variety of gastrointestinal pathogens, including Cryptosporidium, have become available, and these are now widely used. ${ }^{14,15}$ The commercial assays are easy to perform but can be costly. Laboratorydeveloped tests (LDTs), including real-time PCR (RT-PCR) assays, ${ }^{16}$ are less expensive and easily designed, as the genomes for Cryptosporidium hominis ${ }^{17}$ and Cryptosporidium parvum ${ }^{18}$ have been sequenced. However, not all laboratories have the capacity to develop or validate LDTs, and the required instrumentation is expensive. A complicating factor for references laboratories is that samples are received in a variety of fixatives. Thus, the choice of testing method is likely to depend on several factors including available resources, workload, and the population being tested.

As a provider of clinical public health reference testing in New York State, the Wadsworth Center Parasitology Laboratory confirms the presence of Cryptosporidium in patient specimens submitted from hospitals and commercial laboratories. All specimens are evaluated by modified acid-fast staining and direct fluorescent antibody (DFA) immunomicroscopy; however, for approximately $25 \%$ of submitted specimens, Cryptosporidium cannot be identified by microscopy. In this study, we developed a LDT that specifically targets $C$. parvum and $C$. hominis and includes degenerate genus-level primers and a probe to detect other species of Cryptosporidium, which occasionally cause disease in 
humans. Because microscopy was anticipated to be the least sensitive method, the focus was on specimens that were negative when tested by acid-fast staining and immunofluorescent microscopy. After routine analysis, specimens were tested by the LDT as well as a rapid immunoassay for antigen detection and a gastrointestinal panel (GI panel). In addition to the microscopy-negative samples, 20 microscopy-positive specimens were included in the study to verify the specificity of the classical methods. For the purposes of comparison, a composite reference was used such that a specimen was considered a true positive if two or more methods yielded a positive result or the presence of Cryptosporidium could be confirmed by DNA sequencing.

\section{MATERIALS AND METHODS}

Stool sample selection and concentration. Samples were received over a 5-month period from February to March; a total of 92 specimens were tested by all methods. Stool samples were concentrated using Fecal Parasite Concentrator Kits (Evergreen Scientific, Buffalo, NY). Briefly, 1-2 mL of homogenized stool in fixative or transport medium was poured into the provided test tube, and three drops of $20 \%$ Triton X-100 were added along with $1 \mathrm{~mL}$ of CitriSolv (Thermo Fisher Scientific, Hanover Park, IL). The tube was filled with $10 \%$ formalin, the centrifuge tube strainer was screwed in place, and the unit was rocked at $180^{\circ}$ for 30 seconds. Once the liquid portion had all reached the centrifuge tube, then the unit was disassembled, capped, and centrifuged at 1,500 RPM for 10 minutes to pellet the debris and any parasites. The supernatant was poured off, and the pellet was resuspended in a small amount of $10 \%$ formalin. The stool was then applied to clean slides, in an area about the size of a dime, and left to dry overnight in preparation for modified acid-fast and DFA staining.

Staining and microscopy. After fixing the dried specimens in methanol for 10 seconds and air-drying, modified acid-fast staining (Remel, San Diego, CA) was performed as per manufacturer's instructions. Briefly, slides were placed in fuchsin stain for 5 minutes and in decolorizer for 1 second, and counterstained for 4 minutes. Between each step, the slides were rinsed briefly with deionized water. Slides were allowed to air-dry before viewing at $1000 \times$ using bright field microscopy. All of the sample on the slide was reviewed and determined positive if the staining, size, and morphology of organisms present matched with that of a typical Cryptosporidium oocyst.

Direct fluorescent antibody staining was performed on the supplied slides using a Cryptosporidium/Giardia detection kit (Meridian, Cincinnati, $\mathrm{OH}$ ) according to the manufacturer's instructions. One drop of the detection reagent and one drop of the counterstain were added to the dried stool specimen and allowed to incubate for 30 minutes in a humid chamber at room temperature, in the dark. Excess stain was removed from the slide with wash buffer, a drop of mounting medium was added to each sample, and a cover slip was applied to each slide. The entire sample applied to the slide was visualized under 200x magnification using the fluorescein isothiocyanate filter. Samples were determined positive if apple-green staining with the correct pattern and size for Cryptosporidium oocysts were visible.

Stock solution preparation. To determine the limits of detection for the GI panel and the LDT, a stock of $C$. parvum at a concentration of $1 \times 10^{6}$ oocysts/mL (Bunch Grass Farm,
Drury, ID) was spiked into phosphate-buffered saline (PBS) and stool. The stool, which had previously been determined free of all parasites, was preserved in the alcohol-based fixative Total-Fix. One milliliter of the oocyst stock was spiked into $9 \mathrm{~mL}$ of PBS or stool and serially diluted to a concentration of one oocyst $/ \mathrm{mL}$, and then washed and extracted as described in the following text.

DNA extraction. For clinical samples, $1 \mathrm{~mL}$ of homogenized stool in fixative or transport medium was centrifuged in a $1.5-\mathrm{mL}$ tube for 5 minutes at full speed $(20,500 \times g)$, and the supernatant was discarded. The pellet was washed two times with $1 \mathrm{~mL}$ of PBS to remove any residual fixative and resuspended with $1 \mathrm{~mL}$ of NucliSENS easyMAG Lysis Buffer (Biomerieux, Durham, NC). The sample was transferred to Lysing Matrix E tubes (MP Biomedicals, Solon, $\mathrm{OH}$ ), briefly vortexed, and placed on a $75^{\circ} \mathrm{C}$ heat block for 15 minutes. Samples were homogenized in a FastPrep instrument (Thermo Fisher Scientific, Hanover Park, IL) for 45 seconds at speed setting 6.0, and then centrifuged at $20,500 \times g$ for 5 minutes to pellet debris. DNA was extracted using a standard stool extraction protocol on the easyMAG automated DNA extractor (Biomerieux). Briefly, in one vessel, $800 \mu \mathrm{L}$ of stool supernatant, $1.2 \mathrm{~mL}$ NucliSENS Lysis Buffer, 140 $\mu \mathrm{L}$ magnetic silica (Biomerieux), and $10 \mu \mathrm{L}$ of an internal extraction control were combined, extracted using Protocol Specific B 2.0.1, and eluted in $100 \mu \mathrm{L}$.

PCR amplification. Sequences for primers and probes for each PCR reaction are provided in Table 1. Real-time reactions were performed using a QuantaBio 5X ToughMix (QuantaBio, Beverly, MA) containing hot start DNA polymerase, stabilizers, deoxyribonucleotide triphosphates (dNTPs), and $\mathrm{MgCl}_{2}$. All cycling reactions were carried out on a ViiA7 RT-PCR system (Applied Biosystems, Foster City, CA) using the 96 -well block under standard conditions beginning with a 10 -minute $95^{\circ}$ denaturation step followed by 45 cycles of $95^{\circ}$ for 15 seconds and $60^{\circ}$ for 1 minute. A volume of $10 \mu \mathrm{L}$ of extracted DNA was tested in a final reaction volume of $25 \mu \mathrm{L}$; all specimens were tested in duplicate. A second set of duplicate reactions was tested using $1 \mu \mathrm{L}$ of extracted DNA to test for inhibition.

Reactions for nested conventional PCR were performed in duplicate with a final volume of $50 \mu \mathrm{L}$ containing $0.5 \mu \mathrm{M}$ primer, dNTPs at $200 \mu \mathrm{M}$ each, $1 \times$ Phire Reaction Buffer containing $\mathrm{MgCl}_{2}, 1 \mu \mathrm{L}$ of Phire Hot Start II DNA Polymerase (Thermo Scientific), and $10 \mu \mathrm{L}$ of DNA. When the18S rRNA gene was targeted, the conventional thermal cycling conditions were $98^{\circ} \mathrm{C}$ for 30 seconds followed by 35 rounds of $98^{\circ} \mathrm{C}$ for 5 seconds, $55.5^{\circ} \mathrm{C}$ (primary) and $61.6^{\circ} \mathrm{C}$ (secondary) for 5 seconds, $72^{\circ} \mathrm{C}$ for 15 seconds, and a final extension at $72^{\circ} \mathrm{C}$ for 1 minute. When the GP60 gene was targeted, the conventional thermal cycling conditions were $98^{\circ} \mathrm{C}$ for 30 seconds followed by 35 rounds of $98^{\circ} \mathrm{C}$ for 5 seconds, $65.1^{\circ} \mathrm{C}$ (primary) and $54.4^{\circ} \mathrm{C}$ (secondary) for 5 seconds, $72^{\circ} \mathrm{C}$ for 15 seconds, and a final extension at $72^{\circ} \mathrm{C}$ for 1 minute. Products were visualized on a $2 \%$ agarose gel stained with ethidium bromide. Products that were of the expected size (800 bp) were purified using the QIAquick PCR purification kit (Qiagen, Hilden, Germany) according to the manufacturer's instructions before being sequenced by the Wadsworth Center Applied Genomics Technology Cluster.

Positive amplification controls for the RT-PCR assay were designed using genus-level primers and amplifying 1) patient specimens previously determined to be positive for $C$. hominis and Cryptosporidium sp. and 2) C. parvum DNA extracted 
TABLE 1

Primers and probes

\begin{tabular}{|c|c|c|c|c|}
\hline Organism & Type & Primers and probe & Target & Reference \\
\hline $\begin{array}{l}\text { C. parvum and } \\
\text { C. hominis }\end{array}$ & Real-time primers & $\begin{array}{l}\text { F-TTA ATG TAA CTC CAG CTG AAT TCT TTT TC } \\
\text { R-GGA GTT CAG ATT CTT TAA TTT AAT CTA TCA TTT AAT }\end{array}$ & $\begin{array}{l}\text { Gene of unknown } \\
\text { function }\end{array}$ & Ref. 2223 \\
\hline C. hominis & Probe & P-Cy5/ATT TAT CTC TTA CTT CGT GGC GGC G/IBRQ & & \\
\hline C. parvum & Probe & P-TexRd/ATT TAT CTC TTC GTA GCG GCG/BHQ2 & & \\
\hline Crypto. spp & Real-time primers & $\begin{array}{l}\text { F-TGY CCN CCN GGN TTY GTN GAY } \\
\text { R-YGG NGG RCA YTC BGG RTT NGG WGG RGC } \\
\text { P-FAM/CAG GCA TWG/ZEN/TRA AYG CAA CAC AAT CTC } \\
\text { T/IBFQ }\end{array}$ & $\begin{array}{l}\text { Cryptosporidium } \\
\text { oocyst wall } \\
\text { protein }\end{array}$ & This study \\
\hline Crypto. spp. & Conventional (nested) & $\begin{array}{l}\text { F1-TTC TAG AGC TAA TAC ATG CG } \\
\text { R1-CCC ATT TCC TTC GAA ACA GGA } \\
\text { F2-GGA AGG GTT GTA TTT ATT AGA TAA AG } \\
\text { R2-CTC ATA AGG TGC TGA AGG AGT A }\end{array}$ & $18 S$ & Ref. 25 \\
\hline $\begin{array}{l}\text { Crypto. parvum/ } \\
\text { hominis }\end{array}$ & Conventional (nested) & $\begin{array}{l}\text { F1-ATG AGA TTG TCG CTC ATT ATC } \\
\text { R1-TTA CAA CAC GAA TAA GGC TGC } \\
\text { F2-TCC GCT GTA TTC TCA GCC } \\
\text { R2-GGA AGG AAC GAT GTA TCT }\end{array}$ & GP60 & Ref. 2425 \\
\hline
\end{tabular}

from oocysts isolated from cows (Bunch Grass Farm). Control plasmids were constructed using the TOPO TA Cloning Kit for Sequencing (Invitrogen, Carlsbad, CA) following the manufacturer's instructions. Briefly, ligation of appropriate PCR products into the pCR4-TOPO vector was followed by transformation into One Shot competent Escherichia coli cells (Invitrogen). The plasmid was then purified using the QIAGEN Plasmid Mini Kit (Qiagen) and stored at $-20^{\circ} \mathrm{C}$. To test for inhibition, an internal control plasmid ${ }^{19}$ was added to patient specimens after the heating and vortexing step, immediately before automated extraction. In addition, a no template control, which contained no added DNA, a negative extraction control (NEC), and a positive extraction control (PEC) were included in every RT-PCR assay. The PEC included the internal inhibition control plasmid, which was used both to monitor extraction efficiency and as a comparator for amplification from the internal control plasmid added to patient samples. The matrix of the NEC and PEC consisted of previously determined negative stool extracted in tandem with all patient samples to ensure no contamination occurred during handling or DNA extraction.

Commercial GI panel. Samples were tested with a commercially available panel (FilmArray, BioFire, Salt Lake, UT) which includes bacterial, viral, and parasitic pathogens. As the Gl panel is only intended for use with stool samples in CaryBlair transport medium, samples preserved in fixatives were washed as described earlier and resuspended in $1 \mathrm{~mL}$ PBS followed by processing according to the manufacturer's instructions. Briefly, hydration solution was first injected into the pouch, and then, sample buffer and $200 \mu \mathrm{L}$ of sample were added to the sample/buffer injection tube, which was inverted three times and allowed to sit at room temperature for $5 \mathrm{sec}-$ onds before being injected into the pouch. The pouch was loaded into the instrument where the sample undergoes extraction, nucleic acid purification, two rounds of PCR, DNA melt curve analysis, and result interpretation.

Rapid immunoassay assay. All samples were tested using the antigen detection rapid immunoassay Giardia/Cryptosporidium Quik Chek (G/CQC) in accordance with the manufacturer's instructions. Briefly, a preserved stool sample (100 $\mu \mathrm{L}$ ) was added to a tube containing $400 \mu \mathrm{L}$ of diluent and one drop of conjugate. Five hundred microliters of the diluted sample-conjugate mixture were transferred to the sample well of the cartridge and incubated at room temperature for 15 minutes. Wash buffer and two drops of substrate were added to the reaction window, and results were read after 10 minutes.

\section{RESULTS}

Preservative and demographics. Samples were received in a variety of fixatives, as shown in Table 2. Preservation in formalin has been the standard for clinical parasitology, and most specimens were received in either $10 \%$ formalin $(45.7 \%)$ or sodium acetate formalin (SAF) (23.9\%). The age of patients ranged from 10 months to 91 years, although adults aged 22-65 years comprised $43.5 \%$ of the patient population (Table 3). For specimens where the gender of the patient was reported $(86 \%)$, more specimens came from male patients $(60.8 \%)$ than female patients.

Microscopy. Microscopic examination consisted of both modified acid-fast staining and DFA analysis. A sample was considered positive by microscopy if either technique displayed organisms with the staining characteristics and morphological features of Cryptosporidium. Of the 92 samples, microscopy detected 20 positive samples; 19 were positive using both methods and one sample was positive only by modified acid-fast staining. Among the remaining 72 specimens, an additional 15 were positive by two or more methods, and five were positive by one method and confirmed by conventional PCR and sequencing. This resulted in 40 true-positive samples, 51 true-negative samples, and one

TABLE 2

Fixative type for submitted patient samples

\begin{tabular}{lc}
\hline \multicolumn{1}{c}{ Fixative } & Number of samples $(\%)^{\star}$ \\
\hline $10 \%$ formalin & $42(45.7)$ \\
Sodium acetate formalin & $22(23.9)$ \\
Total Fix & $9(9.8)$ \\
Cary-Blair & $4(4.3)$ \\
Polyvinyl alcohol & $2(2.2)$ \\
ProtoFix & $1(1.1)$ \\
ParaPak clean vial & $1(1.1)$ \\
Fixative type not available & $11(12.0)$ \\
\hline${ }^{\star}$ Percentage of 92 samples. &
\end{tabular}


TABLE 3

Age distribution

\begin{tabular}{lc}
\hline Age (years) & Number of specimens $(\%)^{\star}$ \\
\hline $0-1$ & $1(1.1)$ \\
$1-5$ & $16(17.4)$ \\
$6-21$ & $15(16.3)$ \\
$22-65$ & $40(43.5)$ \\
$>65$ & $8(8.7)$ \\
Unknown & $12(13.0)$ \\
\hline${ }^{\star}$ Percentage of 92 samples.
\end{tabular}

specimen for which the status could not be resolved, leaving 91 samples (Figure 1, Supplemental Table 1).

Laboratory-developed test. Because molecular methods have been shown to be more sensitive than microscopy and antigen detection, ${ }^{20}$ we developed an LDT for Cryptosporidium. The LDT was designed to specifically detect $C$. parvum and $C$. hominis, as these species are known to cause most cryptosporidiosis cases. ${ }^{21}$ The assay also was designed to detect Cryptosporidium at the genus level such that infections caused by other species could be detected. For the Cryptosporidium genus-level arm of the assay, the Cryptosporidium oocyst wall protein (COWP) gene was targeted, and primers and probes were designed with degeneracies in the sequence to detect as many species as possible (Supplemental Figure 1). The species-specific primers were designed based on previously published assays and target a gene of unknown function. ${ }^{22,23}$ Forty specimens were positive in the LDT assay, and these were also determined to be positive by the composite reference. As expected, most specimens contained either C. parvum ( $n=24,60 \%)$ or C. hominis $(n=9,22.5 \%)$. Seven specimens (17.5\%) were detected only by the genuslevel COWP portion of the assay. To rule out the possibility that the LDT was incorrectly amplifying DNA from other organisms in DNA extracted from stool samples, alternate primers were used to amplify the $18 \mathrm{~S}$ and GP60 genes of Cryptosporidium for the seven samples detected at the genus level. ${ }^{24,25} \mathrm{Se}-$ quencing of the $18 \mathrm{~S}$ gene identified chipmunk genotype in three specimens, but four could not be identified further. These four samples, however, were confirmed as Cryptosporidium positive using other methods; one of the four samples was positive by all other methods, including microscopy, two were positive by the G/CQC immunoassay, and one was positive by the Gl panel.

Giardia/Cryptosporidium Quick Chek. The G/CQC antigen-based test was able to detect 21 of the $40(52.5 \%)$ true-positive samples and was concordant with all other methods when true negatives were analyzed $(n=51)$. Thus, the sensitivity of the G/CQC compared with the composite reference was $52.5 \%$, and the specificity was $100 \%$. G/CQC

\begin{tabular}{|c|c|c|c|c|c|c|c|c|c|}
\hline & & \multicolumn{2}{|c|}{ Microscopy } & \multicolumn{2}{|c|}{$\begin{array}{c}\text { G/CQC } \\
\text { Antigen } \\
\text { Detection }\end{array}$} & \multicolumn{2}{|c|}{ GI Panel } & \multicolumn{2}{|c|}{$\begin{array}{c}\text { LDT } \\
\text { RT-PCR }\end{array}$} \\
\hline & & Pos & $\mathrm{Neg}$ & Pos & $\mathrm{Neg}$ & Pos & $\mathrm{Neg}$ & Pos & $\mathrm{Neg}$ \\
\hline \multirow{2}{*}{$\begin{array}{l}\text { Composite } \\
\text { Reference }\end{array}$} & $\begin{array}{l}\text { Pos } \\
\text { (40) }\end{array}$ & 20 & 20 & 21 & 19 & 33 & 7 & 40 & 0 \\
\hline & $\begin{array}{l}\mathrm{Neg} \\
(51)\end{array}$ & 0 & 51 & 0 & 51 & 0 & 51 & 0 & 51 \\
\hline
\end{tabular}

FIGURE 1. Results obtained by each method compared with the composite reference. detected Cryptosporidium in five samples that were missed by microscopy but failed to detect the parasite in four specimens that were positive by both modified acid-fast staining and DFA. All of the 19 false-negative specimens were positive when tested by the LDT. Twelve were identified as C. parvum, five as $C$. hominis, and two as other Cryptosporidium species. Thirteen of the samples were also positive in the GI panel, five were confirmed by sequencing, and one was positive via both microscopy methods. When tested using the LDT, samples that were positive by the antigen-based test had a lower average cycle threshold (Ct) value of 28.9 than the false negatives, where the average $\mathrm{Ct}$ value was 32.4 . The G/CQC immunoassay provides optimal results when specimens are tested within 72 hours of collection. The specimens in this study were collected and tested before being submitted to the reference laboratory for confirmation or additional testing. Therefore, the test would not have been performed within that optimal window.

Gastrointestinal panel. The GI panel originally identified 32 of the 40 specimens that were determined to be true positives and all 51 samples that were negative by the composite reference. Testing was repeated on the eight true positives that yielded negative results in the GI panel; of these samples, seven samples again yielded negative results on that platform. One sample gave a positive result on repeat testing. Taking the repeat testing into account, the Gl panel had a sensitivity of $82.5 \%$ and a specificity of $100 \%$ compared with the composite reference.

Looking at the false-negative samples, one was positive by microscopy, by LDT, and by sequencing; and one was positive by both the G/CQC immunoassay and the LDT (Table 4). Five samples were positive by the LDT and confirmed by sequencing to verify the species. All of the specimens with falsenegative results were preserved in $10 \%$ formalin, which is not a validated specimen type for the Gl panel. Interestingly, 17 of the specimens that gave a true-positive result in the Gl panel were also preserved in either $10 \%$ formalin or SAF. Seven specimens that gave positive results with the GI panel were preserved in Total-Fix, four were unpreserved, and for five specimens, the preservative was unknown.

Limits of detection and specificity. The limit of detection (LOD) was determined for both the LDT and GI panel by spiking PBS and stool with known amounts of $C$. parvum oocysts and testing each concentration in duplicate. The overall LOD for the two assays was comparable. When spiked into PBS, both the GI panel and LDT were able to detect Cryptosporidium at a concentration of 100 oocysts $/ \mathrm{mL}$. When oocysts were spiked into stool, the Gl panel detected oocysts at a concentration of 100 oocysts $/ \mathrm{mL}$ and the LDT detected C. parvum oocysts in duplicate at 1,000 oocysts $/ \mathrm{mL}$ and in one of two samples at concentrations of both 100 and 50 oocysts $/ \mathrm{mL}$.

Because $C$. hominis oocysts are not commercially available, the positive-control plasmid bearing the $C$. hominis target was used to test the LOD for this species in the LDT. When spiked into stool, the $C$. hominis plasmid was detected at a concentration of 4,000 copies $/ \mathrm{mL}$. As one oocyst contains four sporozoites, the LODs for C. parvum and C. hominis are comparable. Because the targets of the Gl panel are not known, the LOD for $C$. hominis could not be assessed using that platform.

To evaluate the specificity of the LDT, DNA from a panel of 69 organisms including bacteria, viruses, and other parasites 
TABLE 4

Sequence analysis of discrepant samples

\begin{tabular}{cccc}
\hline Sample & $\begin{array}{c}\text { Gastrointestinal } \\
\text { panel result }\end{array}$ & $\begin{array}{c}\text { Laboratory-developed test real-time } \\
\text { PCR result (average Ct value) }\end{array}$ & Sequence match \\
\hline 2 & $(-)$ & C. parvum (34.3) & C. parvum \\
65 & $(-)$ & C. parvum (40.2) & C. parvum \\
66 & $(-)$ & C. parvum (34.8) & C. parvum \\
77 & $(-)$ & C. parvum (36.0) & C. parvum \\
83 & $(-)$ & C. parvum (29.7) & C. parvum \\
86 & $(-)$ & C. hominis (38.8) & C. hominis \\
87 & $(-)$ & Cryptosporidium sp. (33.9) & NA \\
\hline
\end{tabular}

were evaluated for cross-reactivity (Table 5). Organisms tested included those causing similar gastrointestinal symptoms, pathogens likely to be found in stool specimens, and additional parasites. All samples were negative, except those containing Cryptosporidium.

Analysis of discordant samples. To resolve results for the seven specimens that were positive by LDT and negative by the Gl panel, each was retested by both molecular methods to confirm original results, and the RT-PCR products of the LDT were sequenced to confirm the presence of Cryptosporidium DNA. Six of the seven samples returned sequences confirming the presence of Cryptosporidium DNA. Although the seventh sample did not yield readable sequence, the presence of the target organism was confirmed by a positive result using the antigen-based G/CQC test (Table 4).

\section{DISCUSSION}

Although microscopy has long been the gold standard for the detection of Cryptosporidium, more sensitive molecular-

TABLE 5

Specificity of the laboratory-developed test

\begin{tabular}{lcc}
\hline \multicolumn{1}{c}{ Organism } & Number of species tested & Result \\
\hline Areomonas spp. & 11 & Negative \\
Adenovirus & 1 & Negative \\
Babesia microti & 1 & Negative \\
Blastocystis hominis & 1 & Negative \\
Campylobacter spp. & 3 & Negative \\
Clostridium difficile & 1 & Negative \\
Clostridium sordellii & 1 & Negative \\
Coxsackievirus & 3 & Negative \\
Cryptosporidium hominis & 1 & Positive \\
Cryptosporidium parvum & 1 & Positive \\
Cryptosporidium sp. & 1 & Positive \\
Cyclospora cayetanensis & 1 & Negative \\
Entamoeba histolytica & 1 & Negative \\
Enterovirus & 4 & Negative \\
Escherichia coli & 8 & Negative \\
Giardia lamblia & 1 & Negative \\
HIV & 2 & Negative \\
Leishmania spp. & 2 & Negative \\
Microsporidia & 1 & Negative \\
Norovirus & 2 & Negative \\
Plasmodium spp. & 4 & Negative \\
Plesiomonas shigelloides & 1 & Negative \\
Salmonella enteritidis & 1 & Negative \\
Shigella spp. & 2 & Negative \\
Staphylococcus aureus (Ent A-E) & 5 & Negative \\
Trypanosoma brucei & 1 & Negative \\
Vibrio spp. & 7 & \\
Yersinia enterocolitica & 1 & \\
\hline & & Negative \\
\hline
\end{tabular}

based methods are now available. We tested 91 stool specimens that were submitted to the public health laboratory to confirm the presence of Cryptosporidium. Of those, 20 were positive based on microscopy, another 20 were positive when tested by other methods, and 51 were negative in all assays. Of the 40 samples determined positive by the composite reference, most were found positive by a molecular method and in some cases, by the LDT alone (Figure 2). The LDT described here identified 20 specimens that were negative when tested by microscopy but determined to be positive by the composite reference. The average $\mathrm{Ct}$ value for those specimens when tested by the LDT was 31.7, compared with an average Ct value of 29.2 for the 20 samples that were positive by microscopy. Similarly, as noted earlier, specimens that yielded a false-negative result by $\mathrm{G} / \mathrm{CQC}$ had a higher $\mathrm{Ct}$ value than specimens that were positive by G/CQC. Although the number of samples in each group was low, the results suggest that specimens with a low parasite burden may be missed by the $\mathrm{G} / \mathrm{CQC}$ immunoassay. These results are consistent with previous studies $^{26,27}$ which indicate microscopy and G/CQC lack sensitivity compared with nucleic acid amplificationbased testing.

Although amplification-based testing has better sensitivity, inhibition can be a problem when performing PCR on stool

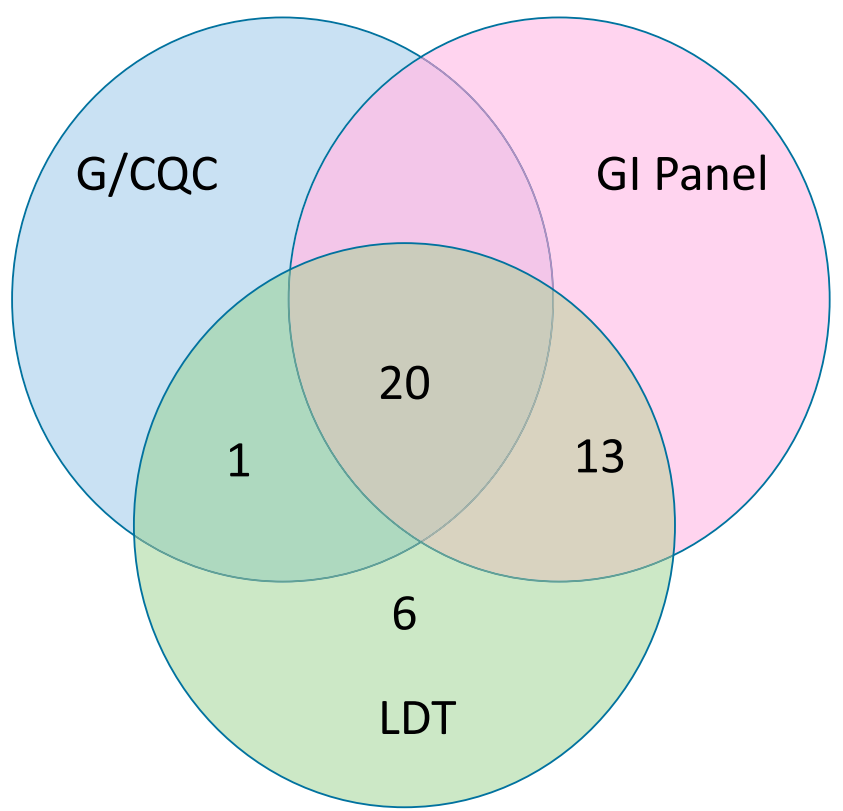

FIGURE 2. Distribution of positive samples by method. 
TABLE 6

Cost of an assay

\begin{tabular}{lc}
\hline \multicolumn{1}{c}{ Detection method } & Approximate cost $(\$)$ \\
\hline Microscopy & 7.50 \\
Giardia/Cryptosporidium Quik Chek & 11.50 \\
Laboratory-developed test & 15.00 \\
Gastrointestinal panel & 130.00 \\
\hline
\end{tabular}

samples, which are inherently variable. In the LDT described here, inhibition was evaluated in two ways. First, the inhibition of sample amplification was monitored by adding an internal inhibition control plasmid to the patient sample, and PEC and Ct values were compared after amplification. Second, for each patient sample, a 1:10 dilution of the extracted DNA was tested to dilute the effect of any possible inhibitors. In two cases, a sample tested at the 1:10 dilution gave duplicate positive results, whereas the undiluted sample gave one positive and one negative result. This indicates that in a small percentage of samples, amplification was inhibited and inhibition could be overcome by diluting the DNA extract.

We also compared the results of the LDT to a commercially available GI panel. All of the samples that yielded discordant results had been preserved in $10 \%$ formalin, which is not approved for this assay. Interestingly, the Gl panel was able to successfully detect Cryptosporidium in 17 samples that had been preserved in a formalin-based fixative. Although the LDT performed well with formalin-fixed specimens in this study, we have observed (data not shown) that the Ct values for formalinfixed stool increased with longer storage times. The results suggest an interference with extraction, inhibition of DNA amplification, or both. Although each formalin-preserved sample processed in this study was washed two times with PBS to remove as much fixative as possible, it is unknown how long each sample was stored in formalin before being washed. Cary-Blair transport medium and preservatives that are alcohol-based are preferred when performing molecular analysis. ${ }^{18}$ The compatibility of these fixatives with molecular analysis was also evident in this study. Both the LDT and the Gl panel produced a positive result for all samples that were submitted in Total-Fix or Cary-Blair transport medium.

For samples that gave discordant results when comparing the LDT and the Gl panel, conventional PCR and sequencing were performed to confirm the presence of Cryptosporidium DNA. For six of the seven samples, sequencing verified the results of the LDT. For one sample, an interpretable sequence could not be obtained; however, the G/CQC antigen-based test was consistent with the presence of Cryptosporidium (Table 4). Of note, this sample had been stored in formalin, potentially contributing to a negative result on the Gl panel and the inability to obtain readable DNA sequence.

Another consideration for reference or clinical laboratories is the cost of performing an assay. In Table 6, we show the cost of each assay, excluding labor. Microscopy and G/CQC are the least expensive, although personnel costs for microscopy can be high as it is labor intensive. Additionally, microscopy and G/CQC were not as sensitive. The LDT is slightly more expensive than microscopy or G/CQC at approximately $\$ 15$ per sample, including DNA extraction and PCR. The LDT is affordable even when performed in duplicate, as there is only a modest increase in cost for the additional PCR reagents. The cost of a single Gl panel is approximately eight-fold higher at
$\$ 130$ per sample, at the time of study. When comparing personnel costs for the LDT and GI panel, although the sample preparation for the LDT can be time consuming, DNA extraction and amplification can be completed for approximately 45 samples in an 8-hour workday. By comparison, the Gl panel requires less hands-on specimen preparation time. However, a single instrument has the maximum capacity of processing one sample at a time and is limited to roughly $7-8$ samples in a day.

Some limitations of this study were due solely to the study being performed by a public health reference laboratory. For example, the optimal time to perform an antigen-based test is within 72 hours of collection. At a reference laboratory, this is not always feasible, as some submitters may hold and batch samples to send multiple specimens to the laboratory at once. Also, the fixative used to collect a sample may not be compatible with the testing algorithm the laboratory has established. Molecular methods are most compatible with a fixative that is ethanol-based or with a specimen that is unpreserved. However, most stool samples for parasitology testing are still collected in a formalin-based fixative, which does not allow for optimal performance of the LDT or the Gl panel. In addition, to properly perform DFA microscopy analysis, a polyvinyl alcohol fixative is not recommended because of the high levels of background that can be generated. As a reference laboratory, we must adapt and perform each test to the best of our capabilities given the specimens we receive.

The LDT described here is a method that laboratories can use to accurately and reliably test for Cryptosporidium in patient stool samples. It has proven to be a sensitive, specific, and costefficient option that allows for fast and easy result interpretation. In addition, the LDT can be used in a high-throughput situation, such as an outbreak. The assay specifically detects $C$. parvum and $C$. hominis. Other species can be detected by the genuslevel primers, although the sensitivity is decreased because of degeneracy in the primers and probes. An added benefit of the LDT is that the extracted DNA can be genotyped to aid in source tracking and epidemiological investigations.

Received October 24, 2019. Accepted for publication April 11, 2020. Published online May 26, 2020.

Note: Supplemental figure and table appear at www.ajtmh.org.

Acknowledgment: We thank the Wadsworth Center Applied Genomics Technology Cluster for performing all sequencing.

Authors' addresses: Kimberly Mergen, Noel Espina, and Allen Teal, Parasitology Laboratory, Wadsworth Center, NYSDOH, Albany, NY, E-mails: kimberly.mergen@health.ny.gov, noel.espina@health.ny.gov, and allen.teal@health.ny.gov. Susan Madison-Antenucci, Parasitology Laboratory, Wadsworth Center, NYSDOH, Albany, NY, and School of Public Health, Biomedical Sciences, University at Albany, Albany, NY, E-mail: s.antenucci@health.ny.gov.

\section{REFERENCES}

1. Katsumata T, Hosea D, Ranuh IG, Uga S, Yanagi T, Kohno S, 2000. Short report: possible Cryptosporidium muris infection in humans. Am J Trop Med Hyg 62: 70-72.

2. Chappell CL, Okhuysen PC, Langer-Curry R, Widmer G, Akiyoshi DE, TanriverdiS, TziporiS, 2006. Cryptosporidium hominis: experimental challenge of healthy adults. Am J Trop Med Hyg 75: 851-857.

3. Adams DA, Thomas KR, Jajosky RA, Foster L, Sharp P, Onweh DH, Schley AW, Anderson WJ; Nationally Notifiable Infectious Conditions G, 2016. Summary of notifiable infectious diseases 
and conditions - United States, 2014. MMWRMorb Mortal Wkly Rep 63: 1-152.

4. Scallan E, Hoekstra RM, Angulo FJ, Tauxe RV, Widdowson MA, Roy SL, Jones JL, Griffin PM, 2011. Foodborne illness acquired in the United States-major pathogens. Emerg Infect Dis 17: 7-15.

5. Painter JE, Hlavsa MC, Collier SA, Xiao L, Yoder JS; CDC, 2015. Cryptosporidiosis surveillance-United States, 2011-2012. MMWR 64: 1-14.

6. Yoder JS et al.; CDC, 2008. Surveillance for waterborne disease and outbreaks associated with recreational water use and other aquatic facility-associated health events-United States, 2005-2006. MMWR Surveill Summ 57: 1-29.

7. CDC, 2012. Promotion of healthy swimming after a statewide outbreak of cryptosporidiosis associated with recreational water venues--Utah, 2008-2009. MMWR Morb Mortal Wkly Rep 61: 348-352.

8. Cantey PT et al., 2012. Outbreak of cryptosporidiosis associated with a man-made chlorinated lake-Tarrant county, Texas, 2008. J Environ Health 75: 14-19.

9. DeSilva MB et al., 2016. Communitywide cryptosporidiosis outbreak associated with a surface water-supplied municipal water system-Baker city, Oregon, 2013. Epidemiol Infect 144: 274-284.

10. Robinson TJ, Cebelinski EA, Taylor C, Smith KE, 2010. Evaluation of the positive predictive value of rapid assays used by clinical laboratories in Minnesota for the diagnosis of cryptosporidiosis. Clin Infect Dis 50: e53-e55.

11. Minak J, Kabir M, Mahmud I, Liu Y, Liu L, Haque R, Petri WA Jr., 2012. Evaluation of rapid antigen point-of-care tests for detection of giardia and Cryptosporidium species in human fecal specimens. J Clin Microbiol 50: 154-156.

12. Alexander CL, Niebel M, Jones B, 2013. The rapid detection of Cryptosporidium and Giardia species in clinical stools using the quik chek immunoassay. Parasitol Int 62: 552-553.

13. Roellig DM et al., 2017. Community laboratory testing for Cryptosporidium: multicenter study retesting public health surveillance stool samples positive for Cryptosporidium by rapid cartridge assay with direct fluorescent antibody testing. PLOS One 12: e0169915.

14. Buss SN, Leber A, Chapin K, Fey PD, Bankowski MJ, Jones MK, Rogatcheva M, Kanack KJ, Bourzac KM, 2015. Multicenter evaluation of the BioFire FilmArray gastrointestinal panel for etiologic diagnosis of infectious gastroenteritis. J Clin Microbiol 53: 915-925.

15. Madison-Antenucci S et al., 2016. Multicenter evaluation of BD max enteric parasite real-time PCR assay for detection of Giardia duodenalis, Cryptosporidium hominis, Cryptosporidium parvum, and Entamoeba histolytica. J Clin Microbiol 54: 2681-2688.

16. Mary C et al., ANOFEL Cryptosporidium National Network. 2013. Multicentric evaluation of a new real-time PCR assay for quantification of Cryptosporidium spp. and identification of Cryptosporidium parvum and Cryptosporidium hominis. J Clin Microbiol 51: 2556-2563.

17. Xu P et al., 2004. The genome of Cryptosporidium hominis. Nature 431: 1107-1112.

18. Abrahamsen MS et al., 2004. Complete genome sequence of the apicomplexan, Cryptosporidium parvum. Science 304: 441-445.

19. Teal AE, Habura A, Ennis J, Keithly JS, Madison-Antenucci S, 2012. A new real-time PCR assay for improved detection of the parasite Babesia microti. J Clin Microbiol 50: 903-908.

20. Elsafi SH, Al-Maqati TN, Hussein MI, Adam AA, Hassan MM, Al Zahrani EM, 2013. Comparison of microscopy, rapid immunoassay, and molecular techniques for the detection of Giardia lamblia and Cryptosporidium parvum. Parasitol Res 112: 1641-1646.

21. Ryan U, Fayer R, Xiao L, 2014. Cryptosporidium species in humans and animals: current understanding and research needs. Parasitology 141: 1667-1685.

22. Hadfield SJ, Robinson G, Elwin K, Chalmers RM, 2011. Detection and differentiation of Cryptosporidium spp. in human clinical samples by use of real-time PCR. J Clin Microbiol 49: 918-924.

23. Jothikumar N, da Silva AJ, Moura I, Qvarnstrom Y, Hill VR, 2008. Detection and differentiation of Cryptosporidium hominis and Cryptosporidium parvum by dual TaqMan assays. J Med Microbiol 57: 1099-1105.

24. Alves M, Xiao L, Sulaiman I, Lal AA, Matos O, Antunes F, 2003. Subgenotype analysis of Cryptosporidium isolates from humans, cattle, and zoo ruminants in Portugal. J Clin Microbiol 41: 2744-2747.

25. Fayer R, Xiao L, 2008. Cryptosporidium and Cryptosporidiosis. Boca Raton, FL: CRC Press; IWA Publishing.

26. Laude $A$ et al., 2016. Is real-time PCR-based diagnosis similar in performance to routine parasitological examination for the identification of Giardia intestinalis, Cryptosporidium parvum/ Cryptosporidium hominis and Entamoeba histolytica from stool samples? Evaluation of a new commercial multiplex PCR assay and literature review. Clin Microbiol Infect 22: 190 e1-190 e8.

27. ten Hove RJ, van Esbroeck M, Vervoort T, van den Ende J, van Lieshout L, Verweij JJ, 2009. Molecular diagnostics of intestinal parasites in returning travellers. Eur J Clin Microbiol Infect Dis 28: 1045-1053.

28. CDC, DPDX, 2016. Stool Specimens - Molecular Diagnosis. Available at: https://www.cdc.gov/dpdx/diagnosticprocedures/stool/ moleculardx.html. Accessed 2019. 